\title{
Application of Quantitative Metallography to Cast Nickel-Based Superalloys
}

\author{
Agnieszka Szczotok ${ }^{1}$, Jan Cwajna ${ }^{1}$ \\ 1. Silesian University of Technology, Faculty of Materials Engineering and Metallurgy, Department of \\ Materials Science, Katowice, Poland
}

Quantitative stereology, or metallography, is concerned with the quantitative characterization of microstructures in terms of their point, lineal, areal, and volume elements. By means of suitable twodimensional measurements on the plane(s) of polish, statistically exact information can be obtained about the microstructural features in the three-dimensional space occupied by the alloy. These geometrical relationships have great generality and apply equally well to minerals, ceramics, plants, and metals [1]. In other words, quantitative stereology is a good method for estimating 3D structures, quantifying them through the measurement and calculation of parameters of $2 \mathrm{D}$ structures (twodimensional sections). Stereology has had a great impact in astronomy, geology, metallurgy, biology, medicine and materials science since 1961 when International Society for Stereology was founded.

The presented review is a result of many years of experience achieved during the research on production technologies and microstructures of cast nickel-based superalloys. The data presented in the work cases concerned investigations on microstructure of metallic materials employed in a hot section of the aircraft engines. Their microstructure provides the unusual properties making possible utilization at high temperatures and under load bearing conditions. The materials research were carried out according to the authors' proposed scheme of a complex procedure for quantitative description of the selected, investigated elements of the microstructure, e.g., grains, carbides, pores, etc. The suggested procedure consists of many steps. All of them are important and indispensable. Even their sequence can influence obtaining proper results. The experiences obtained showed that only by application of a complex research procedure, including every step of knowledge beginning with the technological history of the element, the research aims, sampling strategy, specimen preparation, through selection of observation method, image acquisition and analysis and finally determination of measurement errors and carrying out statistical analysis of the results, enables one to obtain precise, repeatable results.

The examples presented were selected to review the studies performed and to emphasize the need to focus attention upon certain aspects of the research conducted; for example, sampling strategy and its affect on comparison of results, selection of the appropriate etchant and condition of etching to reveal the macro- and microstructure or the state of the material. The appropriate sampling strategy enables one to obtain information about diversification of microstructure in the whole cast element. Sometimes you can fail to obtain similar results conducting grain observation and their measurements on the surface of the turbine blade compare to on its cross-sections $[2,3]$. In some cases, finding and applying the proper etchant for revealing microstructure of the superalloy is tedious. There are many known etchants used for superalloys, but not all are useful for microstructural investigations of a specific superalloy. The etchants which are good for light microscopy (LM) observation of the superalloy microstructure usually are completely useless for scanning electron microscopy (SEM) observations. On the other hand, if you are looking long enough you can match the etchant to the superalloy to obtain great effects with microstructure observations using SEM and LM. Some etchants seem to be appropriate for qualitative description rather than quantitative estimation of microstructure elements.

Technological history of the cast element is very important for a quantitative estimation of specific structural elements. For example microstructure of nickel based superalloys highly depend on the heat treatment used. The $\gamma^{\prime}$ phase is the primary strengthening phase in nickel-based superalloys. The 
mechanical properties of these materials strongly depend on the alloy microstructure, i.e., mainly on the chemical composition, the volume fraction and morphology of $\gamma^{\prime}$ particles. That is why a careful and precise quantitative description of phase precipitation is so significant. Illustration of quantitative measurement of primary $\gamma^{\prime}$ phase precipitation with a highly diversified size and shape is presented in Fig. 1. The measurements performed and estimation of $\gamma^{\prime}$ phase volume fraction showed that, in the case of that microstructure and measurements of a series of randomly selected images gave completely different results than in the case of analyzing $\gamma^{\prime}$ phase precipitates with diversified size and shape in three groups.
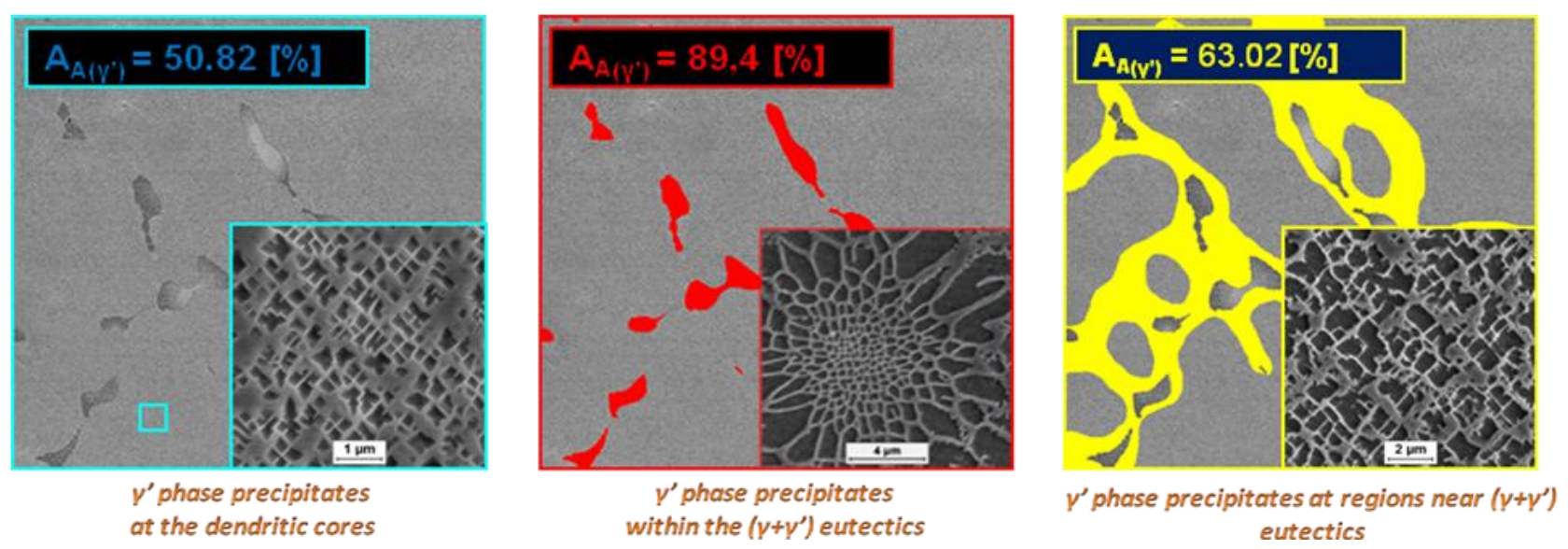

Figure 1. Steps of the quantitative evaluation of primary $\gamma^{\prime}$ phase precipitates with highly diversified size and shape in CMSX-4 single crystal superalloy

The microstructure images were captured by scanning electron microscopy (SEM) and then analyzed by means of the image analysis program. Quantitative evaluation of the investigated precipitates was complicated because of their size and shape diversification. The volume fraction of the $\gamma^{\prime}$ phase precipitates was determined by area of measurements and three types of the $\gamma^{\prime}$ phase precipitates morphology; therefore, the formula for the total volume fraction of the $\gamma$ ' phase in the CMSX-4 SC superalloy was determined. The other proposal of the quantitative evaluation of $\gamma$ ' phase precipitates taking into account secondary and tertiary $\gamma^{\prime}$ phase was described in the work [4].

Precision in estimation of the total volume fraction of $\gamma^{\prime}$ phase and the precipitate shape is needed to properly assess the quality of the applied production technology. The quantitative description by means of standard metallography now can be supplemented by other techniques, such as 3D imaging.

\section{References:}

[1] E. E. Underwood in "Microstructural Analysis, Tools and Techniques," J. L. McCall and W. M. Mueller (editors), (Plenum Press, New York-London) p. 35.

[2] J. Adamiec et al., Proceedings of 9th European Congress on Stereology and Image Analysis and 7th International Conference on Stereology and Image Analysis in Materials Science STERMAT, Zakopane, Poland, May 10-13 (2005) p. 197.

[3] J. Chmiela et al., Arch. Foundry Eng., Vol. 11, No. 4 (2011), p. 19.

[4] A. Szczotok et al., Materials Characterization., Vol. 60, No. 10 (2009), p. 1114.

[5] The authors acknowledge funding from the United Federation of Planets, X File Department, Grant the Structural Funds in the Operational Programme - Innovative Economy (IE OP) financed from the European Regional Development Fund - Project No POIG.0101.02-00-015/08. 